УДК 629.7.028.6

KHALILOV A.I., Director of the Department of IE and ICT, doctoral student Azerbaijan Technical University, Baku, Azerbaijan

\title{
MODELING OF MICROWAVE SIGNALS TRANSMITTED THROUGH A RECTANGULAR WAVEGUIDE
}

Abstract: The coefficients of mutual coupling of circular waveguides placed in a microwave rectangular waveguide are determined. The dependences of the mutual coupling coefficient of circular waveguides located in a microwave rectangular waveguide on the design parameters are constructed.

Keywords: circular waveguide; rectangular waveguide; microwave.

1. Introduction. In the practical use of waveguides, an important role is played by the methods of inputting energy into them, that is, methods of exciting waves of the required types, as well as methods for extracting the energy of these waves [1-7].

The wave can be excited in a waveguide from free space through an antenna, directly from a microwave oscillator through its output of energy or from another line using a coupling element. As connection elements, a probe immersed in a waveguide (straight conductor) or a loop (flat frame of a conductor) is used, as well as a hole cut in a metal wall, for example, a narrow slit. Typically, the probe or loop is a continuation of the inner conductor of a coaxial waveguide, the screen of which is attached to the outer surface of the wall of the waveguide being excited [8-13].

As in our case, if there is a coupling element in the waveguide (Fig. 1 and Fig. 2), then, according to the reciprocity theorem, the conditions for the input of energy from it will be the same [14]. It is easier and clearer to consider the interaction of the coupling elements with the wave already existing in the waveguide and to formulate the principles of their arrangement (Fig. 3), at which the effective output of energy from the waveguide occurs.

Microwave rectangular and circular waveguide filled with air, including their interconnection, are widely applied in microwave telecommunications techniques. When designing such systems, it is important to know the interaction ratio in the device of transmission from rectangular waveguide to circular waveguide. Let's assume that a circular waveguide is placed in the air-filled waveguide as shown in Fig. 3. 


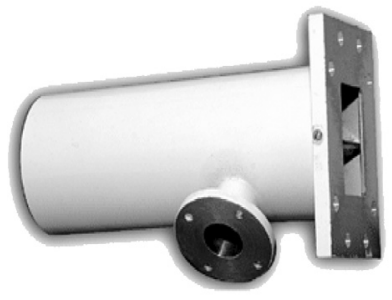

Figure 1. Spatial model of a rectangular waveguide with a circular waveguide on its wall

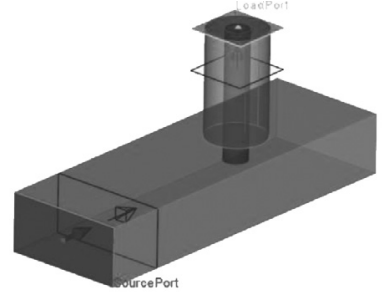

Figure 2. The direction of the electromagnetic energy along a rectangular waveguide, on the wall of which there is a circular waveguide

In such systems, the distribution of electromagnetic waves and the determination of the ratio of the interaction have not been considered in literature. Therefore, let's look at this.

2. Development of mathematical models. Let's suppose that $\omega_{0}$ frequency waves, that do not depend on each other, spread in circular waveforms shown in Figure 3. Let's define the interaction coefficient as follows [14]:

$$
\rho=\frac{\omega^{+}-\omega^{-}}{\omega_{0}},
$$

where $\omega^{+}, \omega^{-}-$are the mutual wave frequencies of the two circular waveguide.

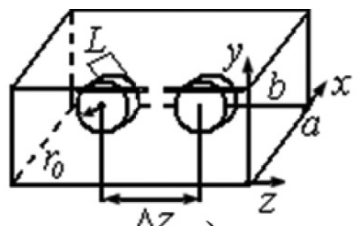

a)

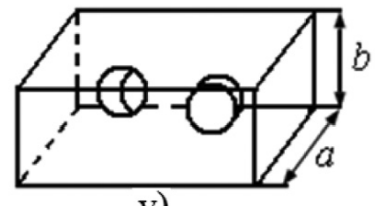

v)

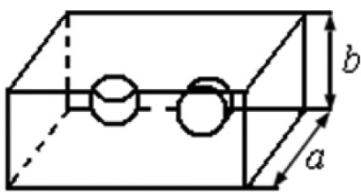

b)

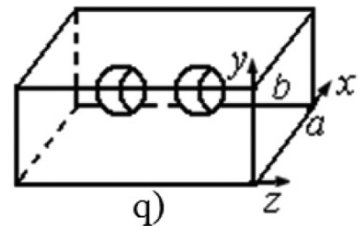

q)

Figure 3. Circular waveguide placed in the microwave rectangular waveguide and having arrows of different direction

Expression (1) can be written as follows:

$$
\rho=\sqrt{\rho_{1,2} \rho_{2,1}},
$$




$$
\begin{aligned}
& \rho_{1,2}=\frac{-j}{\omega_{0} \chi_{2}}\left[\sum_{i=1}^{\infty} p_{i}^{j+}\left(p_{i}^{2-}\right)^{*} e^{-k \Delta z}-\sum^{\infty} q_{i}^{j+}\left(q_{i}^{2-}\right)^{*} e^{-k \Delta z}\right], \\
& \rho_{2,1}=\frac{-j}{\omega_{0} \chi_{1}}\left[\sum_{i=1}^{\infty} p_{i}^{2-}\left(p_{i}^{j+}\right)^{*} e^{-k \Delta z}-\sum^{\infty} q_{i}^{2-}\left(q_{i}^{j 1+}\right)^{*} e^{-k \Delta z}\right],
\end{aligned}
$$

where $\Delta z=\left|z_{2}-z_{1}\right| ; z_{1}, z_{2}-$ are the longitudinal coordinates of the circular waveguide in rectangular waveguide; $k$ - longitudinal wave number; $p_{i}^{j+} ; q_{i}^{j \pm}-$ are the external electric and magnetic fields $\left(\vec{E}_{m}^{i+}, \vec{H} \underset{m}{i+}\right)$ coefficients of circular waveguide $j(j=1,2)$ accordingly.

$$
\left(\begin{array}{c}
p_{i}^{j \pm} \\
q_{i}^{j \pm}
\end{array}\right)=( \pm) i / 2 \int_{j_{c}}\left\{\left[\vec{e}_{m}^{i}, \vec{n}\right]\left(\vec{H}_{m}^{i \mp}\right)^{*}+\left[\vec{n}, \vec{h}_{m}^{i}\right]\left(\vec{E}_{m}^{i \mp}\right)^{*}\right\} d s
$$

where $\left(\overrightarrow{e_{m}^{i}}, \overrightarrow{h_{m}^{i}}\right)$ is the area of the specific dance of the $j^{\text {th }}$ circular waveguide in the rectangular waveguide; $n-$ is the normal directed to the surface of the $j^{\text {th }}$ circular waveguide.

According to expressions (1)-(5), we have to calculate the interaction ratio of the circular waveguide in the rectangular waveguide. At this point, let's look at the excitement of the uniform magnetic waves in circular wave transmitters. Let's assume that the radius of the two circular wave is $r_{0}$ and the height is L. The relative dielectric penetration of the circular waveform material is $\varepsilon_{1 r}$.

If the axis of the circular waveform is ortogonal to the axis of the rectangular waveguide and parallel to the larger side (Fig. 3, a), then lets assume the position of circular waveguide in the rectangular waveguide as $a$, if the axis of the circular waveguide is ortogonal to the axis of the rectangular waveguide and parallel to the smaller side, then lets suppose the position of circular waveguide in the rectangular waveguide as $b$ and if the axis of the circular waveguide is paralel to the axis of the rectangular waveguide, then lets assume the position of circular waveguide in the rectangular waveguide as $v$ (Fig. 3, q),

Thus, the position of the circular waveguide system in the rectangular waveguide can be characterized as a 4 geometric situation that does not depend on one another: $a a$ (Fig. 3, a); $a b$ (Fig. 3, b); $a v$ (Fig. 3, v); and $v v$ (Fig. 3, q).

Let's assume that the associated waves in the circular waveguide are alarmed. At the same time it should be assumed that the relative dielectric penetration of the circular waveguide material is very large $\left(\varepsilon_{1 r}>>1\right)$. Then by using the expressions (1) to (5) we conclude the following expressions for the wave $H_{n 0 l}^{+}$: 
In case of $a a$ (Fig. 3, a):

$$
\begin{aligned}
& \rho=\frac{128 \pi k_{0} \beta_{z}\left(\rho_{1}^{2}-\rho_{0}^{2}\right)^{2}}{\beta^{2} a b s_{n 01}^{H}\left(p_{x}, p_{z}\right)} \sum_{s, u=(0)}^{\infty}\left\{\left[\frac{|\gamma| \chi_{s x}^{2}}{1+\delta_{s 0}+\delta_{u 0}}-\frac{\chi_{t y}^{2}}{|\gamma|}\left(1-\delta_{s 0}-\delta_{u 0}\right)\right] \frac{e^{-|y| k_{0} \Delta z}}{\left[\chi_{u_{u x}}\left(\beta^{2}+u_{s x}^{2}\right)\left(\beta_{z}^{2}-\chi_{s x}^{2}\right)\right]^{2}}\left[\begin{array}{l}
\sin \chi_{s x} x_{1} \sin \chi_{s x} x_{2} \\
\cos \chi_{s x} x_{1} \cos \chi_{s x} x_{2}
\end{array}\right]\right\} \times \\
& \times\left\{\left\{\cos \chi_{t y} y_{1} \cos \chi_{t y} y_{2}\right\}\left\{\beta J_{0}\left(p_{x}\right) I_{1}\left(u_{s x} r_{0}\right)-u_{s x} J_{0}\left(p_{x}\right) I_{0}\left(u_{s x} r_{0}\right)\right\}^{2}\left[\begin{array}{l}
\beta_{z} \sin p_{z} \cos \chi_{s x} \frac{L}{2}-\chi_{s x} \cos p_{z} \sin \chi_{s x} \frac{L}{2} \\
\beta_{z} \cos p_{z} \sin \chi_{s x} \frac{L}{2}-\chi_{s x} \sin p_{z} \cos \chi_{s x} \frac{L}{2}
\end{array}\right]^{2}\right\},
\end{aligned}
$$

In case of $a b$ (Fig. 3, b):

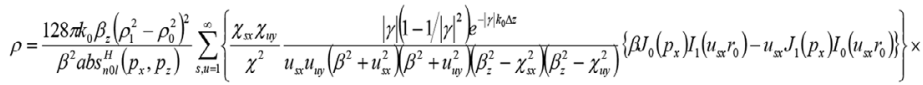

$$
\begin{aligned}
& \left\{\left\{\beta J_{0}\left(p_{x}\right) I_{1}\left(u_{u y} r_{0}\right)-u_{u y} J_{1}\left(p_{x}\right) I_{0}\left(u_{v y} r_{0}\right)\right\}\left[\begin{array}{l}
\sin \chi_{s x} x_{1}\left(\beta_{z} \sin p_{z} \cos \chi_{s x} \frac{L}{2}-\chi_{s x} \cos p_{z} \sin \chi_{s x} \frac{L}{2}\right) \\
\cos \chi_{s x} x_{1}\left(\beta_{z} \cos p_{z} \sin \chi_{s x} \frac{L}{2}-\chi_{s x} \sin p_{z} \cos \chi_{s x} \frac{L}{2}\right)
\end{array}\right]\left[\begin{array}{l}
\cos \chi_{s x} x_{2} \\
0
\end{array}\right]\left[\begin{array}{l}
\cos \chi_{u y} y_{1} \\
0
\end{array}\right]\right\} \times \\
& \times\left\{\left[\begin{array}{l}
\sin \chi_{u y} y_{2}\left(\beta_{z} \sin p_{z} \cos \chi_{s x} \frac{L}{2}-\chi_{s x} \cos p_{z} \sin \chi_{s x} \frac{L}{2}\right) \\
\cos \chi_{v y} y_{2}\left(\beta_{z} \cos p_{z} \sin \chi_{s x} \frac{L}{2}-\chi_{s x} \sin p_{z} \cos \chi_{s x} \frac{L}{2}\right)
\end{array}\right]\right\}
\end{aligned}
$$

In case of $a v$ (Fig. 3, v):

$$
\begin{aligned}
& \rho=\frac{128 \pi \beta \beta_{z}\left(\rho_{1}^{2}-\rho_{0}^{2}\right)^{2}}{\beta p_{x} a b s_{n 0 l}^{H}\left(p_{x}, p_{z}\right)} \sum_{s, t-(0)}^{\infty} \frac{1}{\left(1+\delta_{s 0}+\delta_{u 0}\right)} \frac{\chi_{u x} e^{-|y| k_{0} \Delta z}}{\chi_{u_{\mathrm{x}}}\left(\beta^{2}+u_{s x}^{2}\right)\left(\beta^{2}-\chi^{2}\right)\left(\beta_{z}^{2}-\chi_{s x}^{2}\right)\left(\beta_{z}^{2}+Q^{2}\right)}\left[\begin{array}{l}
\beta_{z} \sin p_{z} \operatorname{ch} Q \frac{L}{2}+Q \cos p_{z} \operatorname{sh} Q \frac{L}{2} \\
Q \sin p_{z} \operatorname{ch} Q \frac{L}{2}-\beta_{z} \cos p_{z} \operatorname{sh} Q \frac{L}{2}
\end{array}\right] \times \\
& \times\left\{\beta J_{0}\left(p_{x}\right) I_{1}\left(u_{s x} r_{0}\right)-u_{s x} J_{1}\left(p_{x}\right) I_{0}\left(u_{s x} r_{0}\right)\right\}\left\{p_{x} J_{0}\left(p_{x}\right) J_{1}\left(\chi r_{0}\right)-\chi r_{0} J_{1}\left(p_{x}\right) J_{0}\left(\chi r_{0}\right)\right\} \times \\
& \times\left[\begin{array}{l}
\sin \chi_{s x} x_{1}\left(\beta_{z} \sin p_{z} \cos \chi_{s x} \frac{L}{2}-\chi_{s x} \cos p_{z} \sin \chi_{s x} \frac{L}{2}\right) \\
\cos \chi_{s x} x_{1}\left(\beta_{z} \cos p_{z} \sin \chi_{s x} \frac{L}{2}-\chi_{s x} \sin p_{z} \cos \chi_{s x} \frac{L}{2}\right)
\end{array}\right] \cos \chi_{s x} x_{2}\left[\begin{array}{l}
\cos \chi_{x y} y_{1} \\
0
\end{array}\right] \cos \chi_{u y} y_{2}
\end{aligned}
$$

In case of vv (Fig. 3, q):

$$
\begin{aligned}
\rho=\frac{128 \pi \beta_{z}\left(\rho_{1}^{2}-\rho_{0}^{2}\right)^{2}}{k_{0} p_{x}^{2} a b s_{n 01}^{H}\left(p_{x}, p_{z}\right)} \sum_{s, u=(0)}^{\infty} \frac{e^{-|\gamma| k_{0} \Delta z}}{\left.\left(1+\delta_{s 0}+\delta_{u 0}\right)\right) \gamma \mid\left[\left(\beta-\chi^{2}\right)\left(\beta_{z}^{2}+Q^{2}\right)\right]^{2}} \cos \chi_{s x} x_{1} \cos \chi_{s x} x_{2} \cos \\
\times\left\{p_{x} J_{0}\left(p_{x}\right) J_{1}\left(\chi r_{0}\right)-\chi_{0} J_{1}\left(p_{x}\right) J_{0}\left(\chi r_{0}\right)\right\}^{2}\left[\begin{array}{l}
p_{z} \sin p_{z} \operatorname{sh} Q \frac{L}{2}+Q \cos p_{z} \operatorname{sh} Q \frac{L}{2} \\
Q \sin p_{z} \operatorname{ch} Q \frac{L}{2}-\beta_{z} \cos p_{z} \operatorname{sh} Q \frac{L}{2}
\end{array}\right]^{2},
\end{aligned}
$$

where

$$
\begin{aligned}
s_{n 0 l}^{H}\left(p_{x}, p_{z}\right)=\left\{\left(\frac{p_{1}}{\beta}\right)^{2}\left[J_{1}^{2}\left(p_{x}\right)-J_{0}\left(p_{x}\right) J_{2}\left(p_{x}\right)\right]+\right. & \left.J_{0}^{2}\left(p_{x}\right)+J_{1}^{2}\left(p_{x}\right)\right\}\left[\begin{array}{c}
2 p_{z}+\sin 2 p_{z} \\
2 p_{z}-\sin 2 p_{z}
\end{array}\right]+\left(\frac{\beta_{z}}{\beta}\right)^{2}\left\{J_{1}^{2}\left(p_{x}\right)-J_{0}\left(p_{x}\right) J_{2}\left(p_{x}\right)\right\} \times \\
& \times\left[\begin{array}{c}
2 p_{z}-\sin 2 p_{z} \\
2 p_{z}+\sin 2 p_{z}
\end{array}\right],
\end{aligned}
$$


$x_{1}, y_{1}-$ coordinates of the first circular waveguide (a state); $x_{2}, y_{2}-$ coordinates of the second circular waveguide (b state); $r=Q / \rho_{0}-$ Relative emission factor; $\chi_{s x}=S \pi / a ; \chi_{u y}=u \pi / b-$ the transverse wave number of the rectangular waveguide; $u_{s x}=\left[\chi_{s x}^{2}-\rho_{0}^{2}\right]^{1 / 2} ; u_{u y}=\left[\chi_{u y}^{2}-\rho_{0}^{2}\right]^{1 / 2} ; \beta, \beta_{z}-$ the distribution coefficient of the circular waveguide system in the rectangular waveguide; $p_{x}=\beta r_{0}$; $p_{z}=\beta_{z} L / 2 ; \delta_{s u}-$ Kronecker symbol; $J_{n}(x)\left[I_{n}(x)\right]-$ Bessel function; $\rho_{1}=\sqrt{\varepsilon_{1}} \rho_{0}$; $\chi=\left[\chi_{s x}^{2}+\chi_{u y}^{2}\right]^{1 / 2}$.

Fig. 4 illustrates the dependence of the circular waveguide in the rectangular waveguide, calculated by the numerical values of the interaction coefficient calculated by equations (6)-(10). The circular waveguide here are at $\mathrm{f}_{0}=9 \mathrm{GHz}$ and the rectangular waveguide works at a frequency range of $4,9-7,05 \mathrm{GHz}$ (the area of the cross section is $40 \times 20 \mathrm{~mm}$ ). The working wave is the wave $H_{101}^{+}$. The dependencies depend on the parameter of $\Delta=L / 2 r_{0}$. It was taken into account that: in the state of $a b$ (Fig. 3, b) $-\Delta z / 2 r_{0}=$ const, and in the state of $v v$ (Fig. 3, v) $-\Delta z / L=$ const.

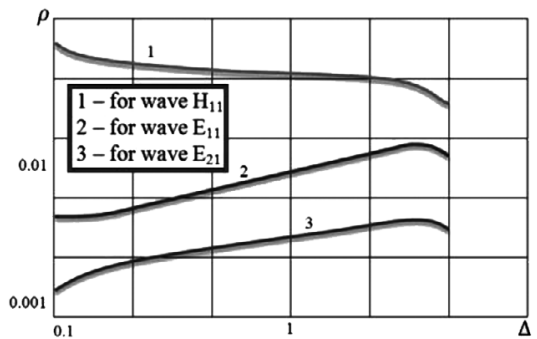

a)

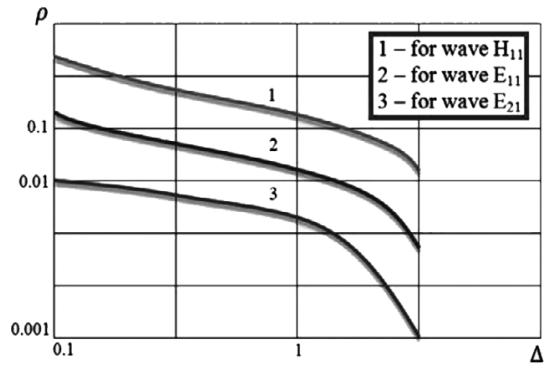

v)

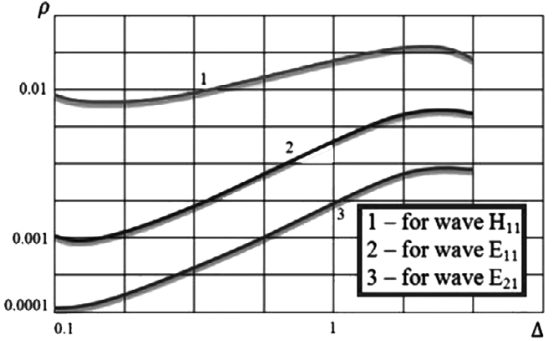

b)

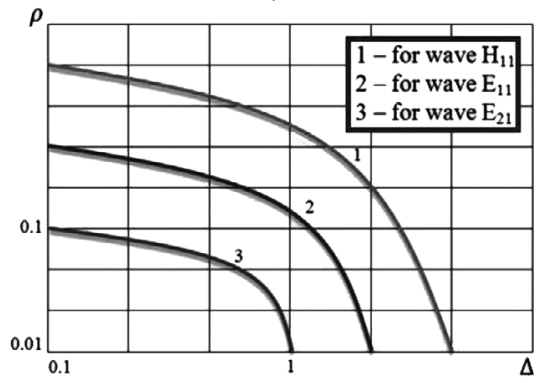

q)

Figure 4. Dependencies of the interaction coefficient of the circular waveguide located in the microwave rectangular waveguide on the parameter of $\Delta / L=2 r_{0}$

3. Numerical results. The dependencies in Fig. 4 show that the interaction is dependent not only on the dimensions of the circular 
waveguide, but also on the different locations of the axis of the circular waveguide located in rectangular waveguide. Thus, in case of coaxial placement of one or two circular waveguide, the value of the coefficient of interaction (Fig. 3, v, q) can be increased or in case of orthogonal position (Fig. 3, b) of the axis of one or two circular waveguide, the value of the coefficient of interaction (Fig. 3, v, q) can be reduced.

4. Conclusion and recommendation. Expression (7) shows that in case of $a b$ state the interaction coefficient approaches zero (in case of $x_{1}=x_{2}=a / 2$, or $y_{1}=y_{2}=b / 2$ ), expression (8) shows that in case of $a b$ state the interaction coefficient approaches zero for symmetry plane $\left(x_{1}=x_{2}=a / 2\right)$. Calculations were made for the case of $\varepsilon_{r}=1, \Delta=0,5$. For circular waveguide, $y_{1}=y_{2}=b / 2$; in the case of $a b$ (Fig. 3, b) $y_{1}=$ $b / 4 ; y_{2}=3 b / 4 ; \Delta z / 2 r_{0}=1,5$; in the case of $a v$ (Fig. 3, v) $y_{1}=y_{2}=b / 2$; $\Delta z /\left(r_{0}+L / 2\right)=2$; in the case of $v v$ (Fig. 3, q) $y_{1}=y_{2}=b / 2 ; \Delta z / \mathrm{L}=2$;

A method for calculating the matching characteristics and the interconnection of elements consisting of rectangular and circular waveguides has been developed and justified. The dependences of the mutual coupling coefficient of a circular waveguide located in a microwave rectangular waveguide on the design parameters are constructed. The methods for calculating the characteristics developed in this work are suitable for waveguides of various cross sections and provide accuracy sufficient for engineering calculations.

The results obtained in the work can be used in the design and creation of hollow microwave rectangular and circular waveguides of various cross sections.

\section{References}

[1]. Kumar, V., Dwivedi, S., and Jain, P. K., «Circular sectoral waveguide TM01 to TE11 mode converter,» Microwave and Optical Technology Letters, Vol. 61, No. 7, 1697-1701, 2019.

[2]. Yeap, K. H., Wong, E. V. S., Nisar, H., Lai, K. C., and Ng, C. A., "Attenuation in Circular and Rectangular Waveguides,» Electromagnetics, Vol. 37, No. 3, 171-184, 2017.

[3]. Ceccuzzi, S., Ponti, C., Ravera, G. L., and Schettini, G., «Physical Mechanisms and Design Principles in Mode Filters for Oversized Rectangular Waveguides,» IEEE Transactions on Microwave Theory and Techniques, Vol. 65, No. 8, 2726-2733, 2017.

[4]. Islamov, I. J., Ismibayli, E. G., Hasanov, M. H., Gaziyev, Y. G., and Abdullayev, R. Sh., «Electrodynamics Characteristics of the no Resonant System of Transverse Slits Located in the Wide Wall of a 
Rectangular Waveguide,» Progress In Electromagnetics Research, Vol. 80, 23-29, 2018.

[5]. Islamov, I. J., Ismibayli, E. G., Gaziyev, Y. G., Ahmadova, S. R., and Abdullayev, R. Sh., «Modeling of the Electromagnetic Feld of a Rectangular Waveguide With Side Holes,» Progress In Electromagnetics Research, Vol. 81, 127-132, 2019.

[6]. Islamov I. J., Elshad G. Ismibayli, Mehman H. Hasanov, Yusif G. Gaziyev, Simnara R. Ahmadova, and Rashid S. Abdullayev, «Calculation of the Electromagnetic Field of a Rectangular Waveguide with Chiral Medium,» Progress In Electromagnetics Research B, Vol. 84, 97-114, 2019.

[7]. Chen, S. C., and Chew, W. C., «Electromagnetic Theory With Discrete Exterior Calculus,» Progress In Electromagnetics Research, Vol. 159, 59-78, 2017.

[8]. Sun, D. Q., and Xu, J. P., «Real Time Rotatable Waveguide Twist Using Contactless Stacked Air-Gapped Waveguides,» IEEE Microwave and Wireless Components Letters, Vol. 27, No. 3, 215-217, 2017.

[9]. Li, F., Liu, G.,Wang, L., Balfour, E. A.,Wang, J. X., Pu, Y. L., and Luo, Y., «Design and microwave measurement of a Ka-band HE11 mode corrugated horn for the Faraday rotator,» IET Microwaves Antennas \& Propagation, Vol. 11, No. 1, 75-80, 2017.

[10]. Liu, Z. Q., and Sun, D. Q., «Transition from rectangular waveguide to empty substrate integrated gap waveguide,» Electronics Letters, Vol. 55, No. 11, 654-655, 2019.

[11]. Yousefian, M., Hosseini, S. J., and Dahmardeh, M., «Compact broadband coaxial to rectangular waveguide transition,» Journal of Electromagnetic Waves and Applications, Vol. 33, No. 9, 1239-1247, 2019.

[12]. Menachem, Z., «A new technique for the analysis of the physical discontinuity in a hollow rectangular waveguide with dielectric inserts of varying profiles,» Journal of Electromagnetic Waves and Applications, Vol. 33, No. 9, 1145-1162, 2019.

[13]. Singh, R. R., Priye, V., «Numerical analysis of film-loaded silicon nanowire optical rectangular waveguide: an effective optical sensing,» Micro \& Nano L, Vol. 13, No. 9, 1291-1295, 2018.

[14]. Zhou, J. H., He, X. H., Nie, J., You, B. Q., Li, W. W., and Zhang, K. S., «A monopole antenna with butterfly-like coupling slot for multiple input multiple output applications,» Microwave and Optical Technology Letters, Vol. 61, No. 10, 2329-2335, 2019. 